Tohoku Math. J.

54 (2002), 239-257

\title{
LIMITING EQUATIONS AND SOME STABILITY PROPERTIES FOR ASYMPTOTICALLY ALMOST PERIODIC FUNCTIONAL DIFFERENTIAL EQUATIONS WITH INFINITE DELAY
}

\author{
YOSHIYUKi Hino* AND SATORU MURAKAMI ${ }^{\dagger}$
}

(Received June 5, 2000, revised January 15, 2001)

\begin{abstract}
For asymptotically almost periodic functional differential equations with infinite delay in a Banach space, some stability properties of a bounded solution are deduced from stabilities in a certain limiting equation which is obtained by employing the Bohr topology.
\end{abstract}

1. Introduction. In this paper we are concerned with an asymptotically almost periodic functional differential equation with infinite delay

$$
\frac{d u}{d t}=A u(t)+F\left(t, u_{t}\right)
$$

on a phase space $\mathcal{B}=\mathcal{B}((-\infty, 0] ; X)$ which possesses a fading memory property, where $X$ is a Banach space and $u_{t}$ is an element belonging to $\mathcal{B}((-\infty, 0] ; X)$ defined by $u_{t}(s)=u(t+s)$ for $s \in(-\infty, 0]$. For ordinary differential equations, D'Anna [4] has shown that the total stability of a bounded solution can be deduced from the total stability in a certain limiting equation which is obtained by employing the Bohr topology. As an example in [3] shows, this result is false when the limiting equations are obtained by using the compact open topology. For functional differential equations on a uniform fading memory space $\mathcal{B}$ with $X=R^{n}$, Hino and Yoshizawa [9] obtained a generalization of D'Anna's result for $\mathcal{B}$-total stability, together with the result for $\mathcal{B}$-uniform asymptotic stability. The $\mathcal{B}$-stability means that the solution remains small if the initial function is small with respect to the semi-norm $|\cdot| \mathcal{B}$. On the one hand, as pointed out in [2], some integrodifferential equations can be set up as functional differential equations on a fading memory space (not uniform) and BC-stability is more practical, where $\mathrm{BC}$-stability means that the solution remains small if the initial function is small with respect to the BC-norm, that is, $\sup _{-\infty<\theta \leq 0}|\phi(\theta)|$.

The main purpose of this paper is to derive $\mathrm{BC}$-stability properties and $\mathcal{B}$-stability properties of a bounded solution of $(\mathrm{E})$ on a fading memory space $\mathcal{B}((-\infty, 0] ; X)$ with a general Banach space $X$ and to extend some results due to D'Anna [4], together with those due to

\footnotetext{
2000 Mathematics Subject Classification. Primary 34K14; Secondary 34K30, 35B15, 35B35.

* Partly supported by the Grant-in-Aid for Scientific Research (C), No.12640155, The Ministry of Education, Science, Sports and Culture, Japan.

$\dagger$ Partly supported by the Grant-in-Aid for Scientific Research (C), No.11640191, The Ministry of Education, Science, Sports and Culture, Japan.
} 
Hino and Yoshizawa [9] for functional differential equations with infinite delay on a uniform fading memory space $\mathcal{B}$ in case of $X=R^{n}$.

The authors wish to thank the referee for his suggestions which are useful for the improvement of the paper.

2. Fading memory spaces and preparatory results. Let $X$ be a Banach space with norm $|\cdot|_{X}$. For any interval $J \subset R:=(-\infty, \infty)$, we denote by $\mathrm{BC}(J ; X)$ the space of all bounded and continuous functions mapping $J$ into $X$. Clearly, $\mathrm{BC}(J ; X)$ is a Banach space with the norm $|\cdot|_{\mathrm{BC}(J ; X)}$ defined by $|\phi|_{\mathrm{BC}(J ; X)}=\sup \left\{|\phi(t)|_{X}: t \in J\right\}$. We often write $|\cdot|_{\mathrm{BC}(J ; X)}$ as $|\cdot|_{J}$. Also, if $J=R^{-}:=(-\infty, 0]$, then we simply write $\mathrm{BC}(J ; X)$ and $|\cdot|_{\mathrm{BC}(J ; X)}$ as $\mathrm{BC}$ and $|\cdot|_{\mathrm{BC}}$, respectively. For any function $u:(-\infty, a) \mapsto X$ and $t<a$, we define a function $u_{t}: R^{-} \mapsto X$ by $u_{t}(s)=u(t+s)$ for $s \in R^{-}$. Let $\mathcal{B}=\mathcal{B}\left(R^{-} ; X\right)$ be a real linear space of functions mapping $R^{-}$into $X$ with a complete seminorm $|\cdot|_{\mathcal{B}}$. The space $\mathcal{B}$ is assumed to have the following properties:

(A1) There exist a positive constant $N$ and locally bounded functions $K(\cdot)$ and $M(\cdot)$ on $R^{+}:=[0, \infty)$ with the property that if $u:(-\infty, a) \mapsto X$ is continuous on $[\sigma, a)$ with $u_{\sigma} \in \mathcal{B}$ for some $\sigma<a$, then for all $t \in[\sigma, a)$,

(i) $u_{t} \in \mathcal{B}$,

(ii) $u_{t}$ is continuous in $t$ (w.r.t. $|\cdot| \mathcal{B}$ ), and

(iii) $N|u(t)|_{X} \leq\left|u_{t}\right|_{\mathcal{B}} \leq K(t-\sigma) \sup _{\sigma \leq s \leq t}|u(s)|_{X}+M(t-\sigma)\left|u_{\sigma}\right|_{\mathcal{B}}$.

(A2) If $\left\{\phi^{k}\right\}$ is a sequence in $\mathcal{B} \cap \mathrm{BC}$ converging to a function $\phi$ uniformly on any compact intertval in $R^{-}$and $\sup _{k}\left|\phi^{k}\right|_{\mathrm{BC}}<\infty$, then $\phi \in \mathcal{B}$ and $\left|\phi^{k}-\phi\right|_{\mathcal{B}} \rightarrow 0$ as $k \rightarrow \infty$.

It is known [7, Proposition 7.1.1] that the space $\mathcal{B}$ contains $\mathrm{BC}$ and that there is a constant $l>0$ such that

$$
|\phi|_{\mathcal{B}} \leq l|\phi|_{\mathrm{BC}}, \quad \phi \in \mathrm{BC} .
$$

Set $\mathcal{B}_{0}=\{\phi \in \mathcal{B}: \phi(0)=0\}$, and define an operator $S_{0}(t): \mathcal{B}_{0} \mapsto \mathcal{B}_{0}$ by

$$
\left[S_{0}(t) \phi\right](s)= \begin{cases}\phi(t+s) & \text { if } t+s \leq 0, \\ 0 & \text { if } t+s>0\end{cases}
$$

for each $t \geq 0$. By virtue of (A1), one can see that the family $\left\{S_{0}(t)\right\}_{t \geq 0}$ is a strongly continuous semigroup of bounded linear operators on $\mathcal{B}_{0}$. Moreover, the space $\mathcal{B}$ is assumed to have the following properties:

$$
\begin{array}{ll}
\text { (A3) } & \lim _{t \rightarrow \infty}\left|S_{0}(t) \phi\right|_{\mathcal{B}}=0, \quad \phi \in \mathcal{B}_{0} . \\
\text { (A3') } \lim _{t \rightarrow \infty}\left\|S_{0}(t)\right\|=0 .
\end{array}
$$

Here and hereafter, we denote by $\|\cdot\|$ the operator norm of linear bounded operators. The space $\mathcal{B}$ is called a fading memory space (resp. a uniform fading memory space), if it satisfies (A3) (resp. $\left(\mathrm{A}^{\prime}\right)$ ) in addition to (A1) and (A2). It is obvious that $\mathcal{B}$ is a fading memory space whenever it is a uniform fading memory space. It is known [7, Proposition 7.1.5] that in the case where $\mathcal{B}$ is a fading memory space, the functions $K(\cdot)$ and $M(\cdot)$ in (A1) can be 
chosen as $K(\cdot) \equiv K$ and $M(\cdot) \equiv M$, constants; while in the case where $\mathcal{B}$ is a uniform fading memory space, the functions $K(\cdot)$ and $M(\cdot)$ can be chosen so as to satisfy $K(\cdot) \equiv$ a constant and $M(t) \rightarrow 0$ as $t \rightarrow \infty$.

We provide a typical example of fading memory spaces. Let $g: R^{-} \mapsto[1, \infty)$ be any continuous nonincreasing function such that $g(0)=1$ and $g(s) \rightarrow \infty$ as $s \rightarrow-\infty$. We set

$$
C_{g}^{0}:=C_{g}^{0}(X)=\left\{\phi: R^{-} \mapsto X \text { is continuous with } \lim _{s \rightarrow-\infty}|\phi(s)|_{X} / g(s)=0\right\} .
$$

Then the space $C_{g}^{0}$ equipped with the norm

$$
|\phi|_{g}=\sup _{s \leq 0} \frac{|\phi(s)|_{X}}{g(s)}, \quad \phi \in C_{g}^{0},
$$

is a Banach space and it satisfies (A1)-(A3). Also, the space $C_{g}^{0}$ is separable whenever $X$ is separable. Moreover, one can see that $\left(\mathrm{A} 3^{\prime}\right)$ holds if and only if $\sup \{g(s+t) / g(s): s \leq$ $-t\} \rightarrow 0$ as $t \rightarrow \infty$. Therefore, if $g(s) \equiv e^{-s}$, then the space $C_{g}^{0}$ is a uniform fading memory space. On the other hand, if $g(s)=1+|s|^{k}$ for some $k>0$, then the space $C_{g}^{0}$ is a fading memory space, but not a uniform fading memory space.

For any set $\mathcal{F}$ in $C\left(R^{+} ; X\right)$, where $C\left(R^{+} ; X\right)$ is the set of all continuous functions defined on $R^{+}$with values in $X$, we set

$$
R(\mathcal{F})=\left\{x(t): x \in \mathcal{F}, t \in R^{+}\right\} .
$$

Moreover, for any set $S$ in $\mathcal{B}$, we set

$$
W(S, \mathcal{F})=\left\{x(\cdot): x \in C(R, X), x_{0} \in S,\left.x\right|_{R^{+}} \in \mathcal{F}\right\}
$$

and

$$
V(S, \mathcal{F})=\left\{x_{t}: t \in R^{+}, x \in W(S, \mathcal{F})\right\}
$$

LEMMA 1 ([8, Lemma 1]). Let $\mathcal{B}$ be a fading memory space. If $S$ is a compact subset in $\mathcal{B}$ and if $\mathcal{F}$ is a uniformly equicontinuous set in $C\left(R^{+}, X\right)$ such that the set $R(\mathcal{F})$ is relatively compact in $X$, then the set $V(S, \mathcal{F})$ is relatively compact in $\mathcal{B}$.

Now we consider the following functional differential equation

$$
\frac{d u}{d t}=A u(t)+F\left(t, u_{t}\right)
$$

where $A$ is the infinitesimal generator of a compact semigroup $\{T(t)\}_{t \geq 0}$ of bounded linear operators on $X$ and $F(t, \phi) \in C\left(R^{+} \times \mathcal{B} ; X\right)$. Throughout this paper, we assume the following condition on $F$ :

(H1) For any $c>0$, there is an $L(c)>0$ such that $|F(t, \phi)|_{X} \leq L(c)$ for all $t \in R^{+}$ and $\phi \in \mathcal{B}$ such that $|\phi|_{\mathcal{B}} \leq c$.

By virture of $(\mathrm{H} 1)$, it follows that for any $(\sigma, \phi) \in R^{+} \times \mathcal{B}$, there exists a function $u \in C\left(\left(-\infty, t_{1}\right) ; X\right)$ such that $u_{\sigma}=\phi$ and the following relation holds:

$$
u(t)=T(t-\sigma) \phi(0)+\int_{\sigma}^{t} T(t-s) F\left(s, u_{s}\right) d s, \quad \sigma \leq t<t_{1},
$$


(cf. [5, Theorem 1]). The function $u$ is called the (mild) solution of (2) through $(\sigma, \phi)$ defined on $\left[\sigma, t_{1}\right)$ and denoted by $u(\cdot, \sigma, \phi, F)$. In the above, $t_{1}$ can be taken as $t_{1}=\infty$ if $\sup _{t<t_{1}}|u(t)|_{X}<\infty$ (cf. [5, Corollary 2]).

LEMma 2. Assume that $\mathcal{B}$ is a fading memory space, $\{T(t)\}_{t \geq 0}$ is a compact semigroup and $F^{k}(t, \phi) \in C\left(R^{+} \times \mathcal{B} ; X\right), k \in N$ (N is the set of all positive integers), satisfy Condition (H1) with $L(\cdot)$ which is independent of $k \in N$. Suppose that $\left\{x^{k}(t)\right\}$ is a family of mild solutions of the equation

$$
\frac{d u}{d t}=A u(t)+F^{k}\left(t, u_{t}\right)
$$

having the following properties:

i) For each $k \in N, x^{k}(t)$ satisfies the integral equation

$$
u(t)=T(t-\sigma) u(0)+\int_{\sigma}^{t} T(t-s) F^{k}\left(s, u_{s}\right) d s
$$

for $\sigma:=\sigma_{k} \leq t \leq \tau_{k} \leq \infty$ (here and hereafter, if $\tau_{k}=\infty$, then $\sigma_{k} \leq t \leq \tau_{k}$ is understood as $\sigma_{k} \leq t<\infty$.)

ii) The set $\left\{x_{\sigma_{k}}^{k}: k \in N\right\}$ is relatively compact in $\mathcal{B}$.

iii) There exists a constant $c$ such that

$$
\left|x^{k}(t)\right|_{X} \leq c, \quad k \in N, \sigma_{k} \leq t \leq \tau_{k} .
$$

Then the set $\left\{x_{t}^{k}: k \in N, \sigma_{k} \leq t \leq \tau_{k}\right\}$ is relatively compact in $\mathcal{B}$.

Proof. We shall prove that the set $W:=\left\{x^{k}(t): \sigma_{k} \leq t \leq \tau_{k}, k \in N\right\}$ is relatively compact in $X$. For this purpose, we assert that any sequence $\left\{a_{k}\right\}$ in $W$ contains a convergent subsequence. We consider the case that $a_{k}=x^{k}\left(\mu_{k}\right)$ with $\sigma_{k} \leq \mu_{k} \leq \tau_{k}$ for $k \in N$. Assume that $\liminf _{k \rightarrow \infty}\left(\mu_{k}-\sigma_{k}\right)=0$. Since the set $\left\{x^{k}\left(\sigma_{k}\right): k \in N\right\}$ is relatively compact in $X$ by ii) and (A1)-(iii), it follows that $\sup _{k \in N}\left|T(\tau) x^{k}\left(\sigma_{k}\right)-x^{k}\left(\sigma_{k}\right)\right|_{X} \rightarrow 0$ as $\tau \rightarrow+0$. Hence the sequence $\left\{x^{k}\left(\mu_{k}\right)-x^{k}\left(\sigma_{k}\right)\right\}$ contains a subsequence which converges to 0 because of the inequality

$$
\begin{aligned}
\left|x^{k}\left(\mu_{k}\right)-x^{k}\left(\sigma_{k}\right)\right|_{X} & =\left|T\left(\mu_{k}-\sigma_{k}\right) x^{k}\left(\sigma_{k}\right)+\int_{\sigma_{k}}^{\mu_{k}} T\left(\mu_{k}-s\right) F^{k}\left(s, x_{s}^{k}\right) d s-x^{k}\left(\sigma_{k}\right)\right|_{X} \\
& \leq\left|T\left(\mu_{k}-\sigma_{k}\right) x^{k}\left(\sigma_{k}\right)-x^{k}\left(\sigma_{k}\right)\right|_{X}+\left(\mu_{k}-\sigma_{k}\right) C_{1} L(H),
\end{aligned}
$$

where $C_{1}=\sup \left\{\|T(\tau)\|: 0 \leq \tau \leq \mu_{k}-\sigma_{k}, k \in N\right\}$ and $H=\sup \left\{\left|x_{t}^{k}\right|_{\mathcal{B}}: \sigma_{k} \leq t \leq \tau_{k}, k \in\right.$ $N\}$. This observation leads to the assertion under the assumption $\liminf _{k \rightarrow \infty}\left(\mu_{k}-\sigma_{k}\right)=0$.

Next we shall establish the assertion under the assumption $\liminf _{k \rightarrow \infty}\left(\mu_{k}-\sigma_{k}\right)>0$. Taking a subsequence if necessary, one can assume that $\inf _{k}\left(\mu_{k}-\sigma_{k}\right) \geq \eta$ for some constant 
$\eta>0$. Let $0<v<\min (\eta, 1)$. By virtue of Property $\mathrm{i})$, we get

$$
\begin{aligned}
x^{k}\left(\mu_{k}\right)= & T\left(\mu_{k}-\sigma_{k}\right) x^{k}\left(\sigma_{k}\right)+\int_{\sigma_{k}}^{\mu_{k}} T\left(\mu_{k}-s\right) F^{k}\left(s, x_{s}^{k}\right) d s \\
= & T(\eta)\left[T\left(\mu_{k}-\sigma_{k}-\eta\right) x^{k}\left(\sigma_{k}\right)+\int_{\sigma_{k}}^{\mu_{k}-\eta} T\left(\mu_{k}-\eta-s\right) F^{k}\left(s, x_{s}^{k}\right) d s\right] \\
& +\int_{\mu_{k}-\eta}^{\mu_{k}} T\left(\mu_{k}-s\right) F^{k}\left(s, x_{s}^{k}\right) d s \\
= & T(\eta) x^{k}\left(\mu_{k}-\eta\right)+T(v) \int_{\mu_{k}-\eta}^{\mu_{k}-v} T\left(\mu_{k}-s-v\right) F^{k}\left(s, x_{s}^{k}\right) d s \\
& +\int_{\mu_{k}-v}^{\mu_{k}} T\left(\mu_{k}-s\right) F^{k}\left(s, x_{s}^{k}\right) d s
\end{aligned}
$$

for $k \in N$. The set $Z:=\left\{\int_{\mu_{k}-\eta}^{\mu_{k}-v} T\left(\mu_{k}-s\right) F^{k}\left(s, x_{s}^{k}\right) d s: k \in N\right\}$ is bounded in $X$, and hence $T(v) Z$ is relatively compact in $X$ because of the compactness of the semigroup $\{T(t)\}_{t \geq 0}$. Similarly, one can derive the relative compactness of the set $T(\eta)\left\{x^{k}\left(\mu_{k}-\eta\right): k \in N\right\}$. Thus we have

$$
\begin{aligned}
\alpha\left(\left\{x^{k}\left(\mu_{k}\right): k \in N\right\}\right) & =\alpha\left(\left\{\int_{\mu_{k}-v}^{\mu_{k}} T\left(\mu_{k}-s\right) F^{k}\left(s, x_{s}^{k}\right) d s: k \in N\right\}\right) \\
& \leq v C_{2} L(H),
\end{aligned}
$$

where $C_{2}=\sup _{0 \leq \tau \leq 1}\|T(\tau)\|$ and $\alpha(\cdot)$ is Kuratowski's measure of noncompactness of sets in $X$. For the details of the properties of $\alpha(\cdot)$, see [10, Section 1.4]. It follows that $\alpha\left(\left\{x^{k}\left(\mu_{k}\right)\right.\right.$ : $k \in N\})=0$ because $v$ is arbitrary. Thus the set $\left\{x^{k}\left(\mu_{k}\right): k \in N\right\}$ is relatively compact in $X$, and hence the sequence $\left\{x^{k}\left(\mu_{k}\right)\right\}$ contains a convergent subsequence.

In order to complete the proof of the assertion, it remains only to establish the assertion in the case of $a_{k}=x^{k_{0}}\left(\mu_{k}\right)$ with $\sigma_{k_{0}} \leq \mu_{k} \leq \tau_{k_{0}}$ for $k \in N$ and for some $k_{0} \in N$. Repeating almost the same argument as in the preceding paragraph, we can see that the set $\left\{x^{k_{0}}(t)\right.$ : $\left.\sigma_{k_{0}} \leq t \leq \tau_{k_{0}}\right\}$ is relatively compact in $X$, and hence the sequence $\left\{a_{k}\right\}$ contains a convergent subsequence, as required.

Let $\sigma_{k} \leq s \leq t \leq \min \left(\tau_{k}, s+1\right)$. Then

$$
x^{k}(t)=T(t-s) x^{k}(s)+\int_{s}^{t} T(t-\tau) F^{k}\left(\tau, x_{\tau}^{k}\right) d \tau,
$$

and hence

$$
\begin{aligned}
\left|x^{k}(t)-x^{k}(s)\right|_{X} & \leq\left|T(t-s) x^{k}(s)-x^{k}(s)\right|_{X}+\left|\int_{s}^{t} T(t-s) F^{k}\left(\tau, x_{\tau}^{k}\right) d \tau\right|_{X} \\
& \leq \sup \left\{|T(t-s) z-z|_{X}: z \in W\right\}+L(H) C_{2}|t-s| .
\end{aligned}
$$

Since $W$ is relatively compact in $X, T(\tau) z$ is uniformly continuous in $\tau \in[0,1]$ uniformly for $z \in W$. This observation leads to $\sup _{k \in N}\left|x^{k}(t)-x^{k}(s)\right|_{X} \rightarrow 0$ as $|t-s| \rightarrow 0$.

For each $k \in N$, we define a function $y^{k}(t)$ by $y^{k}(t)=x^{k}\left(\sigma_{k}+t\right)$ if $t \leq \tau_{k}-\sigma_{k}$, and $y^{k}(t)=x^{k}\left(\tau_{k}\right)$ if $t>\tau_{k}-\sigma_{k}$. Then $\left\{y^{k}(t): t \in R^{+}, k \in N\right\}=W$ is relatively compact in 
$X$ and the family of functions $\left\{y^{k}(\cdot): k \in N\right\}$ is uniformly equicontinuous on $R^{+}$. Therefore Lemma 1 yields that the set $\left\{y_{t}^{k}: k \in N, t \in R^{+}\right\}$is relatively compact in $\mathcal{B}$. Hence the set $\left\{x_{t}^{k}: k \in N, \sigma_{k} \leq t \leq \tau_{k}\right\}$ is relatively compact in $\mathcal{B}$. This completes the proof of Lemma 2.

3. Limiting equations and asymptotically almost periodic solutions. In the remainder of this paper, we always assume that $\mathcal{B}$ is a separable fading memory space.

A function $F(t, \phi) \in C\left(R^{+} \times \mathcal{B}, X\right)$ is said to be asymptotically almost periodic in $t$ uniformly for $\phi \in \mathcal{B}$, if it is a sum of continuous functions $P(t, \phi)$ and $Q(t, \phi)$ such that $P(t, \phi)$ is almost periodic in $t$ uniformly for $\phi \in \mathcal{B}$ and that $Q(t, \phi) \rightarrow 0$ uniformly for $\phi \in S$ for any compact set $S$ in $\mathcal{B}$ as $t \rightarrow \infty$.

A sequence $\left\{F_{k}\right\}$ in $C\left(R^{+} \times \mathcal{B} ; X\right)$ is said to be convergent to $G$ Bohr-uniformly on $R^{+} \times \mathcal{B}$ if $F_{k}$ converges to $G$ uniformly on $R^{+} \times S$ for any compact set $S$ in $\mathcal{B}$ as $k \rightarrow \infty$. A function $F(t, \phi) \in C\left(R^{+} \times \mathcal{B}, X\right)$ is said to be positively precompact if for any sequence $\left\{t_{k}\right\}$ in $R^{+}$such that $t_{k} \rightarrow \infty$ as $k \rightarrow \infty$, the sequence $\left\{F\left(t+t_{k}, \phi\right)\right\}$ contains a Bohr-uniformly convergent subsequence.

In the case where $F(t, \phi) \equiv F(t)$ and $X=R^{n}$, it is known (e.g. [11, pp. 20-30]) that $F(t, \phi)$ is asymptotically almost periodic in $t$ uniformly for $\phi \in \mathcal{B}$ if and only if $F(t, \phi)$ is positively precompact. In fact, by virtue of the separability for $\mathcal{B}$, we can see that the argument employed in [11, pp. 20-30] to establish the above equivalence works for any $F(t, \phi) \in$ $C\left(R^{+} \times \mathcal{B} ; X\right)$ when $X$ is any (separable) Banach space.

In what follows, we always assume the following conditions on Equation (2) in addition to $(\mathrm{H} 1)$ :

(H2) Equation (2) has a bounded solution $\bar{u}(t)$ defined on $R^{+}$such that $\bar{u}_{0} \in \mathrm{BC}$ and $\sup _{t \in R^{+}}\left|\bar{u}_{t}\right|_{\mathcal{B}}<\infty$.

(H3) $F(t, \phi)$ is asymptotically almost periodic in $t$ uniformly for $\phi \in \mathcal{B}$.

For any $\tau \in R^{+}$, we define the $\tau$-translation $F^{\tau}$ of $F(t, \phi)$ by

$$
F^{\tau}(t, \phi)=F(t+\tau, \phi), \quad(t, \phi) \in R^{+} \times \mathcal{B} .
$$

Clearly, $F^{\tau}$ is in $C\left(R^{+} \times \mathcal{B} ; X\right)$, too. As was explained in the preceding paragraph, (H3) implies that $F(t, \phi)$ is positively precompact. Therefore any sequence $\left\{\tau_{n}^{\prime}\right\} \subset R^{+}$contains a subsequence $\left\{\tau_{n}\right\}$ such that $\left\{F^{\tau_{n}}\right\}$ converges Bohr-uniformly on $R^{+} \times \mathcal{B}$. We set

$$
H(F)=\overline{\left\{F^{\tau}: \tau \in R^{+}\right\}} \text {in } C\left(R^{+} \times \mathcal{B} ; X\right),
$$

where $\overline{\left\{F^{\tau}: \tau \in R^{+}\right\}}$denotes the closure of $\left\{F^{\tau}: \tau \in R^{+}\right\}$in $C\left(R^{+} \times \mathcal{B} ; X\right)$ equipped with the Bohr topology. The set $H(F)$ is called the hull of $F$. Clearly, the hull $H(F)$ is invariant with respect to the $\tau$-translation; that is, $G^{\tau} \in H(F)$ whenever $G \in H(F)$ and $\tau \in R^{+}$. For any $G \in H(F)$, one can choose a sequence $\left\{\tau_{n}\right\} \subset R^{+}$so that $F^{\tau_{n}} \rightarrow G$ Bohr-uniformly on $R^{+} \times \mathcal{B}$ as $n \rightarrow \infty$. In particular, we denote by $\Omega(F)$ the set of all elements $G$ in $H(F)$ for which one can choose a sequence $\left\{\tau_{n}\right\} \subset R^{+}$so that $\left\{\tau_{n}\right\} \rightarrow \infty$ as $n \rightarrow \infty$ and $F^{\tau_{n}} \rightarrow G$ Bohr-uniformly on $R^{+} \times \mathcal{B}$. In fact, by (H3) we can assume that any $G \in \Omega(F)$ is defined on 
$R \times \mathcal{B}$ and almost periodic in $t$ uniformly for $\phi \in \mathcal{B}$. If $G \in H(F)$, then the equation

$$
\frac{d v}{d t}=A v(t)+G\left(t, v_{t}\right) \quad t \in R^{+},
$$

is called an equation in the hull of (2). In particular, if $G \in \Omega(F)$, then it is called a limiting equation of (2).

Under the above assumptions (H1)-(H3), it is known [8] that $O_{\bar{u}, R^{+}}:=\overline{\left\{\bar{u}(t): t \in R^{+}\right\}}$ is compact in $X, \bar{u}(t)$ is uniformly continuous on $R^{+}, X_{\bar{u}, R^{+}}:=\overline{\left\{\bar{u}_{t}: t \in R^{+}\right\}}$is compact in $\mathcal{B}$ and $\bar{u}_{t} \in \mathcal{B}$ is uniformly continuous in $t \in R^{+}$. Therefore, for any sequence $\left\{\tau_{n}^{\prime}\right\} \subset R^{+}$one can choose a subsequence $\left\{\tau_{n}\right\} \subset\left\{\tau_{n}^{\prime}\right\}, \bar{v} \in C(R ; X)$ and $G \in H(F)$ such that $\lim _{n \rightarrow \infty} F^{\tau_{n}}=$ $G$ in $C\left(R^{+} \times \mathcal{B} ; X\right)$ and $\lim _{n \rightarrow \infty}\left|\bar{u}_{t+\tau_{n}}-\bar{v}_{t}\right|_{\mathcal{B}}=0$ uniformly on any compact interval in $R^{+}$. In this case, we write as

$$
\left(\bar{u}^{\tau_{n}}, F^{\tau_{n}}\right) \rightarrow(\bar{v}, G) \text { compactly, }
$$

for simplicity. Denote by $H(\bar{u}, F)$ the set of all $(\bar{v}, G) \in C(R ; X) \times H(F)$ such that $\left(\bar{u}^{\tau_{n}}, F^{\tau_{n}}\right) \rightarrow(\bar{v}, G)$ compactly for some sequence $\left\{\tau_{n}\right\} \subset R^{+}$. In particular, we denote by $\Omega(\bar{u}, F)$ the set of all elements $(\bar{v}, G)$ in $H(\bar{u}, F)$ for which one can choose a sequence $\left\{\tau_{n}\right\} \subset R^{+}$so that $\lim _{n \rightarrow \infty} \tau_{n}=\infty$ and $\left(\bar{u}^{\tau_{n}}, F^{\tau_{n}}\right) \rightarrow(\bar{v}, G)$ compactly. It is known [8] that $\bar{v}$ is a solution of (3) whenever $(\bar{v}, G) \in H(\bar{u}, F)$.

Now we shall give some definitions of BC-stabilities.

Definition 1. The bounded solution $\bar{u}(t)$ of (2) is said to be BC-totally stable (BCTS) if for any $\varepsilon>0$ there exists a $\delta(\varepsilon)>0$ with the property that $\sigma \in R^{+}, \phi \in \mathrm{BC}$ with $\left|\bar{u}_{\sigma}-\phi\right|_{\mathrm{BC}}<\delta(\varepsilon)$ and $h \in \mathrm{BC}([\sigma, \infty) ; X)$ with $\sup _{t \in[\sigma, \infty)}|h(t)|_{X}<\delta(\varepsilon)$ imply $|\bar{u}(t)-u(t, \sigma, \phi, F+h)|_{X}<\varepsilon$ for $t \geq \sigma$.

Definition 2. The bounded solution $\bar{u}(t)$ of (2) is said to be BC-uniformly stable (BC-US) if for any $\varepsilon>0$ there exists a $\delta(\varepsilon)>0$ such that $\sigma \in R^{+}$and $\phi \in \mathrm{BC}$ with $\left|\bar{u}_{\sigma}-\phi\right|_{\mathrm{BC}}<\delta(\varepsilon)$ imply $|\bar{u}(t)-u(t, \sigma, \phi, F)|_{X}<\varepsilon$ for $t \geq \sigma$. Furthermore, $\bar{u}(t)$ is said to be BC-uniformly asymptotically stable (BC-UAS), if it is BC-US and there exists a $\delta_{0}>0$ with the property that for any $\varepsilon>0$ there exists a $t_{0}(\varepsilon)>0$ such that $\sigma \in R^{+}$and $\phi \in \mathrm{BC}$ with $\left|\bar{u}_{\sigma}-\phi\right|_{\mathrm{BC}}<\delta_{0}$ imply $|\bar{u}(t)-u(t, \sigma, \phi, F)|_{X}<\varepsilon$ for $t \geq \sigma+t_{0}(\varepsilon)$.

In the above, we can define $\mathcal{B}$-total stability $(\mathcal{B}$-TS) if we replace " $\phi \in \mathcal{B}$ with $\left|\bar{u}_{\sigma}-\phi\right|_{\mathcal{B}}<\delta(\varepsilon)$ " in place of " $\phi \in \mathrm{BC}$ with $\left|\bar{u}_{\sigma}-\phi\right|_{\mathrm{BC}}<\delta(\varepsilon)$ ". Moreover, we can define $\mathcal{B}$-uniform asymptotic stability (B-UAS) in a similar way.

The following proposition can be obtained by following the argument in [8, Theorem 1].

PROPOSITION 1. If the solution $\bar{u}(t)$ of (2) is $\mathrm{BC}-\mathrm{TS}$, then it is asymptotically almost periodic in $t$.

The following theorem is an extension of Hino and Yoshizawa [9, Theorem 1] to the case where $X$ is a Banach space and $\mathcal{B}$ is a fading memory space.

THEOREM 1. If Equation (2) admits a limiting equation (3) whose solution $\bar{v}(t)$ such that $(\bar{v}, G) \in \Omega(\bar{u}, F)$ is $\mathrm{BC}-\mathrm{TS}$, then $\bar{u}(t)$ is asymptotically almost periodic in $t$. 
Proof. Since $(\bar{v}, G) \in \Omega(\bar{u}, F)$, there is a sequence $\left\{\tau_{k}\right\}, \tau_{k} \rightarrow \infty$ as $k \rightarrow \infty$, such that $\left(\bar{u}^{\tau_{k}}, F^{\tau_{k}}\right) \rightarrow(\bar{v}, G)$ compactly. We shall show that $\bar{u}\left(t+\tau_{k}\right)$ is convergent uniformly on $R^{+}$. Suppose the contrary. Then, for some $\varepsilon>0$ there are sequences $\left\{t_{j}\right\},\left\{k_{j}\right\}$ and $\left\{m_{j}\right\}$ such that

$$
k_{j} \rightarrow \infty \quad \text { and } \quad m_{j} \rightarrow \infty \quad \text { as } j \rightarrow \infty
$$

(4) $\left|\bar{u}\left(\tau_{k_{j}}+t_{j}\right)-\bar{u}\left(\tau_{m_{j}}+t_{j}\right)\right|_{X}=\varepsilon \quad$ and $\quad\left|\bar{u}\left(\tau_{k_{j}}+t\right)-\bar{u}\left(\tau_{m_{j}}+t\right)\right|_{X}<\varepsilon \quad$ on $\left[0, t_{j}\right)$. Put $v^{j}(t)=\bar{u}\left(\tau_{k_{j}}+t\right)$ and $w^{j}(t)=\bar{u}\left(\tau_{m_{j}}+t\right)$. Then we may assume that

$$
\rho\left(v_{0}^{j}, \bar{v}_{0}\right)<\frac{1}{j}
$$

and

$$
\rho\left(w_{0}^{j}, \bar{v}_{0}\right)<\frac{1}{j},
$$

where $\rho(\phi, \psi):=\sum_{l=1}^{\infty} 2^{-l}|\phi-\psi|_{l} /\left\{1+|\phi-\psi|_{l}\right\}$ and $|\cdot|_{l}=|\cdot|_{[-l, 0]}$. For each $j \in N$ and $r \in R^{+}$, we define a function $v^{j, r}: R \mapsto X$ by

$$
v^{j, r}(t)= \begin{cases}v^{j}(t), & -r \leq t, \\ v^{j}(-r)+\bar{v}(t)-\bar{v}(-r), & t<-r\end{cases}
$$

First, we shall show that

$$
\sup \left\{\left|v_{0}^{j, r}-v_{0}^{j}\right|_{\mathcal{B}}: j \in N\right\} \rightarrow 0 \quad \text { as } r \rightarrow \infty .
$$

If this is not the case, then there exist an $\varepsilon_{0}>0$ and sequences $\left\{j_{k}\right\} \subset N$ and $\left\{r_{k}\right\}, r_{k} \rightarrow$ $\infty$ as $k \rightarrow \infty$, such that $\left|v_{0}^{j_{k}, r_{k}}-v_{0}^{j_{k}}\right|_{\mathcal{B}} \geq \varepsilon_{0}$ for $k=1,2, \ldots$ Put $\psi^{k}=v_{0}^{j_{k}, r_{k}}-v_{0}^{j_{k}}$. Clearly, $\left\{\psi^{k}\right\}$ is a sequence in $\mathrm{BC}$ which converges to the zero function uniformly on any compact set in $R^{-}$and $\sup _{k}\left|\psi^{k}\right|_{\mathrm{BC}}<\infty$. Then Axiom (A2) yields that $\left|\psi^{k}\right|_{\mathcal{B}} \rightarrow 0$ as $k \rightarrow \infty$, a contradiction.

Observe that $v^{j, r}(t) \rightarrow \bar{v}(t)$ as $j \rightarrow \infty$, uniformly for $(t, r)$ in any compact set in $R \times R^{+}$. Hence the set $\left\{v_{0}^{j}, \quad v_{0}^{j, r}: j \in N, \quad r \in R^{+}\right\}$is relatively compact in $\mathcal{B}$, because the set $X_{\bar{u}, R^{+}}$is compact in $\mathcal{B}$ and $v_{0}^{j} \in X_{\bar{u}, R^{+}}$. Moreover, the set $\left\{v^{j}(t), v^{j, r}(t): j \in N, r \in\right.$ $\left.R^{+}, t \in R^{+}\right\}$is contained in the compact set $O_{\bar{u}, R^{+}}$. From these observations and Lemma 1 it follows that the set $W:=\left\{v_{t}^{j}, v_{t}^{j, r}: j \in N, r \in R^{+}, t \in R^{+}\right\}$is relatively compact in $\mathcal{B}$. Consequently,

$$
\sup \left\{\left|F\left(t+\tau_{k}, \phi\right)-G(t, \phi)\right|_{X}: t \in R^{+}, \phi \in W\right\} \rightarrow 0 \quad \text { as } k \rightarrow \infty .
$$

Define a continuous function $q^{j, r}$ on $R^{+}$by

$$
q^{j, r}(t)= \begin{cases}F\left(t+\tau_{k_{j}}, v_{t}^{j}\right)-G\left(t,\left(v^{j, r}\right)_{t}\right), & 0 \leq t \leq t_{j}, \\ q^{j, r}\left(t_{j}\right), & t_{j}<t .\end{cases}
$$

Since

$$
\left|\left(v^{j, r}\right)_{t}-\left(v^{j}\right)_{t}\right|_{\mathcal{B}} \leq M\left|v_{0}^{j, r}-v_{0}^{j}\right|_{\mathcal{B}} \quad\left(t \in R^{+}, j \in N\right)
$$


by Axiom (A1), it follows from (6) that

$$
\sup \left\{\left|G\left(t, v_{t}^{j}\right)-G\left(t,\left(v^{j, r}\right)_{t}\right)\right|_{X}: t \in R^{+}, j \in N\right\} \rightarrow 0 \quad \text { as } r \rightarrow \infty .
$$

Hence, by (7) and (8) we can choose $j_{0}:=j_{0}(\varepsilon) \in N$ and $r=r(\varepsilon) \in N$ in such a way that

$$
\sup \left\{\left|q^{j, r}(t)\right|_{X}: j \geq j_{0}, t \in R^{+}\right\}<\delta(\varepsilon / 2) / 2,
$$

where $\delta(\cdot)$ is the one for the BC-TS of the solution $\bar{v}(t)$ of (3). Moreover, for this $r$, select an integer $j \geq j_{0}$ such that $j>2^{r}(1+\delta(\varepsilon / 2)) / \delta(\varepsilon / 2)$. Then $2^{-r}\left|\bar{v}_{0}-v_{0}^{j}\right|_{r} /\left[1+\left|\bar{v}_{0}-v_{0}^{j}\right|_{r}\right] \leq$ $\rho\left(\bar{v}_{0}, v_{0}^{j}\right)<2^{-r} \delta(\varepsilon / 2) /[1+\delta(\varepsilon / 2)]$ by (5), which implies that

$$
\left|\bar{v}_{0}-v_{0}^{j}\right|_{r}<\delta(\varepsilon / 2) \quad \text { or } \quad\left|v_{0}^{j, r}-\bar{v}_{0}\right|_{\mathrm{BC}}<\delta(\varepsilon / 2) .
$$

Since the function $v^{j, r}$ is the solution of

$$
\frac{d v}{d t}=A v(t)+G\left(t, v_{t}\right)+q^{j, r}(t)
$$

for $t \in\left[0, t_{j}\right]$, and since $\bar{v}(t)$ is a BC-TS solution of (3), from the fact that $\sup _{t \geq 0}\left|q^{j, r}(t)\right|<$ $\delta(\varepsilon / 2)$ it follows that $\left|\left(v^{j, r}\right)(t)-\bar{v}(t)\right|_{X}<\varepsilon / 2$ on $\left[0, t_{j}\right]$. In particular, we have $\mid\left(v^{j, r}\right)\left(t_{j}\right)-$ $\left.\bar{v}\left(t_{j}\right)\right|_{X}<\varepsilon / 2$ or $\left|v^{j}\left(t_{j}\right)-\bar{v}\left(t_{j}\right)\right|_{X}<\varepsilon / 2$.

By the same way, we have $\left|w^{j}\left(t_{j}\right)-\bar{v}\left(t_{j}\right)\right|_{X}<\varepsilon / 2$. Thus $\left|v^{j}\left(t_{j}\right)-w^{j}\left(t_{j}\right)\right|_{X} \leq \mid v^{j}\left(t_{j}\right)-$ $\left.\bar{v}\left(t_{j}\right)\right|_{X}+\left|\bar{v}\left(t_{j}\right)-w^{j}\left(t_{j}\right)\right|_{X}<\varepsilon / 2+\varepsilon / 2=\varepsilon$, which contradicts (4). Therefore $\bar{u}\left(t+\tau_{k}\right)$ must converge uniformly on $R^{+}$.

Finally, we shall verify that $\bar{u}(t)$ is asymptotically almost periodic in $t$. For any sequence $\left\{s_{k}^{\prime}\right\}$ such that $s_{k}^{\prime} \rightarrow \infty$ as $k \rightarrow \infty$, one can choose a subsequence $\left\{s_{k}\right\}$ of $\left\{s_{k}^{\prime}\right\}$ so that $s_{k}-\tau_{k}=: \mu_{k} \rightarrow \infty$ as $k \rightarrow \infty$. By virtue of Proposition $1, \bar{v}(t)$ is asymptotically almost periodic in $t$, and hence one can assume that $\bar{v}\left(t+\mu_{k}\right)$ converges uniformly on $R^{+}$. Then $\bar{u}\left(t+s_{k}\right)=\bar{u}\left(t+\tau_{k}+\mu_{k}\right)$ also converges uniformly on $R^{+}$. Therefore $\bar{u}(t)$ is asymptotically almost periodic in $t$. This completes the proof of the theorem.

4. Inherited properties in asymptotically almost periodic functional differential equations. A property for the solution $\bar{u}(t)$ of (2) is called an inherited property, if each solution $\bar{v}(t)$ such that $(\bar{v}, G) \in \Omega(\bar{u}, F)$ also possesses the property. The following result shows that the BC-TS property is an inherited property.

THEOREM 2. If the solution $\bar{u}(t)$ of (2) is BC-TS, then for any $(\bar{v}, G) \in \Omega(\bar{u}, F)$, $\bar{v}(t)$ also is BC-TS with a common pair $\left(\varepsilon, \delta_{1}(\varepsilon)\right)$.

Proof. Let $\rho(\cdot)$ be the one introduced in the proof of Theorem 1 , and let $U:=$ $\left\{x \in X:|x|_{X} \leq c\right\}$ be a closed, bounded subset of $X$ whose interior contains $O_{\bar{u}, R}:=$ $\overline{\{\bar{u}(t): t \in R\}}$. In what follows, we write $\phi(\cdot) \in U$ whenever $\phi \in \mathrm{BC}$ and $\phi(\theta) \in U$ for all $\theta \leq 0$.

We first note that the solution $\bar{u}(t)$ of (2) is BC-TS if and only if the following assertion holds (for the proof, see [6]): 
ASSERTION 1. (*) For any $\varepsilon>0$ there exists a $\delta^{*}(\varepsilon)>0$ with the property that $\sigma \in R^{+}, \phi(\cdot) \in U$ with $\rho\left(\phi, \bar{u}_{\sigma}\right)<\delta^{*}(\varepsilon)$ and $h \in \mathrm{BC}([\sigma, \infty) ; X)$ with $|h|_{[\sigma, \infty)}<\delta^{*}(\varepsilon)$ imply $|u(t, \sigma, \phi, F+h)-\bar{u}(t)|_{X}<\varepsilon$ for $t \geq \sigma$.

Next, we shall certify the following assertion:

ASSERTION 2. Property (*) is an inherited property.

Let $v$ be the solution of

$$
\frac{d v}{d t}=A v(t)+G\left(t, v_{t}\right)+h(t)
$$

through $\left(s, v_{s}\right)$, where $s \geq 0,(\bar{v}, G) \in \Omega(\bar{u}, F)$ and $h(t)$ is a continuous function on $[s, \infty)$. Assume that

$$
\rho\left(\bar{v}_{s}, v_{s}\right)<\delta^{*}(\varepsilon / 2) / 2 \text { and }|h(t)|_{X}<\delta^{*}(\varepsilon / 2) / 2 \text { on }[s, \infty),
$$

where $\varepsilon$ is any positive number satisfying $|x|<c-\varepsilon$ for all $x \in O_{\bar{u}, R}$, and $\delta^{*}(\cdot)$ is the number given in Assertion 1. Suppose that for some $\tau>0$,

$$
|\bar{v}(s+\tau)-v(s+\tau)|_{X}=\varepsilon \quad \text { and } \quad|\bar{v}(t)-v(t)|_{X}<\varepsilon \quad \text { for } s \leq t<s+\tau .
$$

Since $(\bar{v}, G) \in \Omega(\bar{u}, F)$, there exists a sequence $\left\{t_{k}\right\}, t_{k} \rightarrow \infty$ as $k \rightarrow \infty$, such that $\left(\bar{u}^{t_{k}}, F^{t_{k}}\right) \rightarrow(\bar{v}, G)$ compactly. Therefore there is a $k_{0}=k_{0}(\varepsilon)>0$ such that

$$
\rho\left(\bar{u}_{s+\sigma}, \bar{v}_{s}\right)<\delta^{*}(\varepsilon / 2) / 2
$$

and

$$
|F(t+\sigma, \phi)-G(t, \phi)|_{X}<\delta^{*}(\varepsilon / 2) / 2 \text { on }[s, s+\tau] \times\left\{X_{v, R^{+}} \cup X_{\bar{v}, R^{+}}\right\},
$$

where $\sigma=t_{k_{0}}$. Thus there are continuous functions $p(t)$ and $q(t)$ defined on $[s, \infty)$ such that $|p|_{[s, \infty)}<\delta^{*}(\varepsilon / 2),|q|_{[s, \infty)}<\delta^{*}(\varepsilon / 2)$,

$$
p(t)=G\left(t, \bar{v}_{t}\right)-F\left(t+\sigma, \bar{v}_{t}\right) \quad \text { on }[s, s+\tau]
$$

and

$$
q(t)=G\left(t, v_{t}\right)-F\left(t+\sigma, v_{t}\right)+h(t) \quad \text { on }[s, s+\tau] .
$$

Then $\bar{v}(t)$ is a solution of

$$
\frac{d u}{d t}=A u(t)+F\left(t+\sigma, u_{t}\right)+p(t)
$$

on $[s, s+\tau]$, and $v(t)$ is a solution of

$$
\frac{d u}{d t}=A u(t)+F\left(t+\sigma, u_{t}\right)+q(t)
$$

on $[s, s+\tau]$. On the other hand, it is clear that $\bar{u}(t+\sigma)$ is a solution of

$$
\frac{d u}{d t}=A u(t)+F\left(t+\sigma, u_{t}\right) .
$$

Notice that $\bar{v}_{S}(\cdot) \in U$ and $v_{s}(\cdot) \in U$ because of $\bar{v}_{S}(\theta) \in O_{\bar{u}, R}$ for all $\theta \leq 0$. Since $\rho\left(\bar{u}_{s+\sigma}, \bar{v}_{s}\right)<\delta^{*}(\varepsilon / 2)$ and $|p|_{[s, \infty)}<\delta^{*}(\varepsilon / 2)$, it follows from Assertion 1 that

$$
|\bar{u}(t+\sigma)-\bar{v}(t)|_{X}<\varepsilon / 2 \text { for } s \leq t \leq s+\tau .
$$


Moreover, $|q|_{[s, \infty)}<\delta^{*}(\varepsilon / 2)$ and $\rho\left(\bar{u}_{s+\sigma}, v_{s}\right) \leq \rho\left(\bar{u}_{s+\sigma}, \bar{v}_{s}\right)+\rho\left(\bar{v}_{s}, v_{s}\right)<\delta^{*}(\varepsilon / 2)$, and hence we have

$$
|\bar{u}(t+\sigma)-v(t)|_{X}<\varepsilon / 2 \text { for } s \leq t \leq s+\tau .
$$

Thus it follows from (10) and (11) that $|\bar{v}(s+\tau)-v(s+\tau)|_{X}<\varepsilon$, which contradicts (9).

Since $\rho(\phi, \psi) \leq|\phi-\psi|_{\mathrm{BC}}$ for $\phi, \psi \in \mathrm{BC}$, the theorem follows from Assertion 2 . Indeed, we may put $\delta_{1}(\cdot)=\delta^{*}(\cdot / 2) / 2$.

COROLlary 1. Under the assumptions of Theorem 1 , any $\bar{w}(t)$ such that $(\bar{w}, P) \in$ $\Omega(\bar{u}, F)$ is BC-TS with a common pair $\left(\varepsilon, \delta_{1}(\varepsilon)\right)$.

Proof. By Proposition 1 and Theorem $1, \bar{u}(t)$ and $\bar{v}(t)$ are asymptotically almost periodic in $t$, and hence $\Omega(\bar{u}, F)=\Omega(\bar{v}, G)$, because $F(t, \phi)$ is asymptotically almost periodic in $t$ uniformly for $\phi \in \mathcal{B}$. Thus, for any $(\bar{w}, P) \in \Omega(\bar{v}, G), \bar{w}(t)$ is BC-TS with a common pair $\left(\varepsilon, \delta_{1}(\varepsilon)\right)$, which follows by applying Theorem 2 to $(\bar{v}, G)$, since $\bar{v}(t)$ is BC-TS.

5. Stability properties via limiting equations. In this section, we shall study stability properties for the solution $\bar{u}(t)$ of (2) via limiting equations. Before stating our results, we prove a result which gives the continuous dependence property of solutions for the initial value problem in some sense.

We say that the solution $\bar{u}(t)$ of (2) is unique for the initial value problem if $\bar{u}_{\sigma}=\psi$ implies $\bar{u}(t)=u(t, \sigma, \psi, F)$ for all $t \geq \sigma$.

Proposition 2. Assume that the solution $\bar{u}(t)$ of (2) is unique for the initial value problem. Then, for any $\varepsilon>0, \sigma \geq 0$ and $s>0$, there exists $a \delta=\delta(\varepsilon, \sigma, s)>0$ such that if $\left|\bar{u}_{\sigma}-\phi\right|_{\mathcal{B}}<\delta$ and $\sup _{t \in[\sigma, \sigma+s]}|h(t)|_{X}<\delta$, then for any $t \in[\sigma, \sigma+s]$

$$
|\bar{u}(t)-u(t, \sigma, \phi, F+h)|_{X}<\varepsilon,
$$

where $u(t, \sigma, \phi, F+h)$ is a solution of

$$
\frac{d u}{d t}=A u(t)+F\left(t, u_{t}\right)+h(t)
$$

through $(\sigma, \phi)$.

ProOf. Assume that the conclusion does not hold. Then there exist constants $\varepsilon>$ $0, \sigma_{0} \geq 0$ and $s_{0}>0$ and sequences $\left\{\phi^{k}\right\} \in \mathcal{B},\left\{t_{k}\right\},\left\{\tau_{k}\right\} \subset\left[0, s_{0}\right], t_{k}<\tau_{k}$, and $\left\{h^{k}(t)\right\} \in$ $C\left(\left[\sigma_{0}, \sigma_{0}+s_{0}\right] ; X\right), \sup _{t \in\left[\sigma_{0}, \sigma_{0}+s_{0}\right]}\left|h^{k}(t)\right|_{X}<1 / k$, such that

$$
\begin{gathered}
\left|\bar{u}_{\sigma_{0}}-\phi^{k}\right|_{\mathcal{B}}<\frac{1}{k}, \\
\left|\bar{u}\left(t_{k}+\sigma_{0}\right)-x^{k}\left(t_{k}+\sigma_{0}\right)\right|_{X}=\varepsilon, \\
\left|\bar{u}\left(\tau_{k}+\sigma_{0}\right)-x^{k}\left(\tau_{k}+\sigma_{0}\right)\right|_{X}=2 \varepsilon, \\
\left|\bar{u}\left(t+\sigma_{0}\right)-x^{k}\left(t+\sigma_{0}\right)\right|_{X}<\varepsilon \quad\left(0 \leq t<t_{k}\right)
\end{gathered}
$$

and

$$
\left|\bar{u}\left(t+\sigma_{0}\right)-x^{k}\left(t+\sigma_{0}\right)\right|_{X}<2 \varepsilon \quad\left(0 \leq t<\tau_{k}\right)
$$


for $k=1,2, \ldots$, where $x^{k}(t)=u\left(t, \sigma_{0}, \phi^{k}, F+h^{k}\right)$ is a solution of

$$
\frac{d u}{d t}=A u(t)+F\left(t, u_{t}\right)+h^{k}(t)
$$

through $\left(\sigma_{0}, \phi^{k}\right)$. By choosing a subsequence if necessary, we may assume that $t_{k} \rightarrow t_{0} \in$ $\left[0, s_{0}\right]$. Applying Lemma 2 with $F(t, \phi)+h^{k}(t)$ in place of $F^{k}(t, \phi)$, we see that the set $\left\{x_{t}^{k}: k \in N, \sigma_{0} \leq t \leq \sigma_{0}+\tau_{k}\right\}$ is relatively compact in $\mathcal{B}$, and hence the set $V:=\left\{x^{k}(t)\right.$ : $\left.k \in N, \sigma_{0} \leq t \leq \sigma_{0}+\tau_{k}\right\}$ is relatively compact in $X$.

Now we shall show that

$$
\inf \left\{\tau_{k}-t_{k} \mid k=1,2, \ldots\right\}=: 2 a>0 .
$$

If $a=0$, then we may assume that $\tau_{k}-t_{k} \rightarrow 0$, by taking a subsequence. Since $x^{k}(t)$ is a mild solution of (14) through $\left(t_{k}+\sigma_{0}, x_{t_{k}+\sigma_{0}}^{k}\right)$, we get

$$
\begin{aligned}
\left|x^{k}\left(\tau_{k}+\sigma_{0}\right)-x^{k}\left(t_{k}+\sigma_{0}\right)\right|_{X} \\
=\mid T\left(\tau_{k}-t_{k}\right) x^{k}\left(t_{k}+\sigma_{0}\right) \\
\quad+\int_{t_{k}+\sigma_{0}}^{\tau_{k}+\sigma_{0}} T\left(\tau_{k}+\sigma_{0}-s\right)\left\{F\left(s, x_{s}^{k}\right) d s+h^{k}(s)\right\} d s-\left.x^{k}\left(t_{k}+\sigma_{0}\right)\right|_{X} \\
\leq\left|\left(T\left(\tau_{k}-t_{k}\right)-I\right) x^{k}\left(t_{k}+\sigma_{0}\right)\right|_{X} \\
\quad+\left|\int_{t_{k}+\sigma_{0}}^{\tau_{k}+\sigma_{0}} T\left(\tau_{k}+\sigma_{0}-s\right)\left\{F\left(s, x_{s}^{k}\right) d s+h^{k}(s)\right\} d s\right|_{X} \\
\leq \sup _{z \in V}\left|\left(T\left(\tau_{k}-t_{k}\right)-I\right) z\right|_{X}+\left(\tau_{k}-t_{k}\right) C_{1}\left\{L\left(H^{*}\right)+\frac{1}{k}\right\},
\end{aligned}
$$

and hence

$$
\begin{aligned}
\varepsilon= & \left|\bar{u}\left(\tau_{k}+\sigma_{0}\right)-x^{k}\left(\tau_{k}+\sigma_{0}\right)\right|_{X}-\left|\bar{u}\left(t_{k}+\sigma_{0}\right)-x^{k}\left(t_{k}+\sigma_{0}\right)\right|_{X} \\
\leq & \left|\bar{u}\left(\tau_{k}+\sigma_{0}\right)-\bar{u}\left(t_{k}+\sigma_{0}\right)-\left\{x^{k}\left(\tau_{k}+\sigma_{0}\right)-x^{k}\left(t_{k}+\sigma_{0}\right)\right\}\right|_{X} \\
\leq & \left|\bar{u}\left(\tau_{k}+\sigma_{0}\right)-\bar{u}\left(t_{k}+\sigma_{0}\right)\right|_{X}+\left|x^{k}\left(\tau_{k}+\sigma_{0}\right)-x^{k}\left(t_{k}+\sigma_{0}\right)\right|_{X} \\
\leq & \left|\bar{u}\left(\tau_{k}+\sigma_{0}\right)-\bar{u}\left(t_{k}+\sigma_{0}\right)\right|_{X} \\
& +\sup _{z \in V}\left|\left(T\left(\tau_{k}-t_{k}\right)-I\right) z\right|_{X}+\left(\tau_{k}-t_{k}\right) C_{1}\left\{L\left(H^{*}\right)+\frac{1}{k}\right\},
\end{aligned}
$$

where $C_{1}=\sup _{0 \leq \tau \leq s_{0}}\|T(\tau)\|$ and $H^{*}=\sup \left\{\left|x_{t}^{k}\right|_{\mathcal{B}}: \sigma_{0} \leq t \leq \sigma_{0}+\tau_{k}, k \in N\right\}$. The right hand side of the above inequality tends to zero as $k \rightarrow \infty$, because $\lim _{t \rightarrow 0^{+}} T(t) z=z$ uniformly for $z \in V$, which is a contradiction. Thus we may assume that $x^{k}(t)$ exists on $\left[\sigma_{0}, \sigma_{0}+t_{0}+a\right]$ and $\sup _{\sigma_{0} \leq t \leq \sigma_{0}+t_{0}+a}\left|x_{t}^{k}\right|_{\mathcal{B}} \leq H^{*}$.

Since the set $\left\{x^{k}(s): k \in N, s \in\left[\sigma_{0}, \sigma_{0}+t_{0}+a\right]\right\}$ is relatively compact in $X$, by the same reasoning as in the proof of Lemma 2 we see that $\left\{x^{k}(t)\right\}$ is a family of equicontinuous functions on $\left[\sigma_{0}, \sigma_{0}+t_{0}+a\right]$. Hence we may assume that $x^{k}(t)$ converges to some continuous 
function $y(t)$ uniformly on $\left[\sigma_{0}, \sigma_{0}+t_{0}+a\right]$ as $k \rightarrow \infty$. Put $y_{0}=\bar{u}_{\sigma_{0}}$. Since

$$
x^{k}(t)=T\left(t-\sigma_{0}\right) \phi^{k}(0)+\int_{\sigma_{0}}^{t} T(t-s)\left\{F\left(s, x_{s}^{k}\right)+h^{k}(s)\right\} d s
$$

for $t \in\left[\sigma_{0}, \sigma_{0}+t_{0}+a\right]$ and $\left|\phi^{k}(0)-\bar{u}\left(\sigma_{0}\right)\right|_{X} \rightarrow 0(k \rightarrow \infty)$, and since $\left\{x_{t}^{k}: t \in\right.$ $\left.\left[\sigma_{0}, \sigma_{0}+t_{0}+a\right], k \in N\right\}$ is relatively compact in $\mathcal{B}$, it follows from (15) that

$$
y(t)=T\left(t-\sigma_{0}\right) \bar{u}\left(\sigma_{0}\right)+\int_{\sigma_{0}}^{t} T(t-s) F\left(s, y_{s}\right) d s \quad\left(\sigma_{0} \leq t \leq \sigma_{0}+t_{0}+\frac{a}{2}\right) .
$$

Consequently, $y(t)$ is a solution of (2) through $\left(\sigma_{0}, \bar{u}_{\sigma_{0}}\right)$ on $\left[\sigma_{0}, \sigma_{0}+t_{0}+a / 2\right]$, and hence $y(t)=\bar{u}(t)$ on $\left[\sigma_{0}, \sigma_{0}+t_{0}+a / 2\right]$ by the uniqueness of $\bar{u}(t)$ for the initial value problem. On the other hand, letting $k \rightarrow \infty$ in (13), we have

$$
\left|\bar{u}\left(t_{0}+\sigma_{0}\right)-y\left(t_{0}+\sigma_{0}\right)\right|_{X}=\varepsilon,
$$

which is a contradiction. This completes the proof of the proposition.

Remark 1. By virtue of (1) one can see that Proposition 2 holds good, whenever $\left|\bar{u}_{\sigma}-\phi\right|_{\mathcal{B}}<\delta$ can be replaced by $\left|\bar{u}_{\sigma}-\phi\right|_{\mathrm{BC}}<\delta$.

Now we are ready to prove theorems that are generalizations of a result obtained by D'Anna [4] for ordinary differential equations and a result obtained by Hino and Yoshizawa [9] for functional differential equations on a uniform fading memory space $\mathcal{B}$ in the case $X=R^{n}$.

THEOREM 3. Assume that $\mathcal{B}$ is a fading memory space and $\bar{u}(t)$ is unique for the initial value problem. If Equation (2) admits a limiting equation (3) whose solution $\bar{v}(t)$ such that $(\bar{v}, G) \in \Omega(\bar{u}, F)$ is BC-TS, then $\bar{u}(t)$ is BC-TS.

Proof. First of all, we shall show that $\bar{u}(t)$ is eventually BC-TS, that is, for any $\varepsilon>0$ there exist $\alpha(\varepsilon) \geq 0$ and $\delta(\varepsilon)>0$ such that if $s \geq \alpha(\varepsilon),\left|\bar{u}_{s}-\psi\right|_{\mathrm{BC}}<\delta(\varepsilon)$ and $h(t)$ is a continuous function which satisfies $|h(t)|_{X}<\delta(\varepsilon)$ on $[s, \infty)$, then

$$
|\bar{u}(t)-u(t, s, \psi, F+h)|_{X}<\varepsilon \quad \text { for } t \geq s,
$$

where $u(\cdot, s, \psi, F+h)$ is a solution through $(s, \psi)$ of (12).

Suppose that $\bar{u}(t)$ is not eventually BC-TS. Then there exist an $\varepsilon>0$ and sequences $\left\{t_{k}\right\},\left\{r_{k}\right\},\left\{h^{k}(t)\right\},\left\{x^{k}(t)\right\}$ such that $t_{k}>k, r_{k}>t_{k},\left|x_{t_{k}}^{k}-\bar{u}_{t_{k}}\right|_{\mathrm{BC}}<1 / k,\left|h^{k}(t)\right|<1 / k$ on $\left[t_{k}, \infty\right),\left|x^{k}\left(r_{k}\right)-\bar{u}\left(r_{k}\right)\right|_{X}=\varepsilon$ and $\left|x^{k}(t)-\bar{u}(t)\right|_{X}<\varepsilon$ on $\left[t_{k}, r_{k}\right)$, where $h^{k}(t)$ is a continuous function and $x^{k}(t)$ is a solution through $\left(t_{k}, x_{t_{k}}^{k}\right)$ of

$$
\frac{d u}{d t}=A u(t)+F\left(t, u_{t}\right)+h^{k}(t) .
$$

Then there is an $s_{k}, t_{k}<s_{k}<r_{k}$, such that

$$
\left|x_{s_{k}}^{k}-\bar{u}_{s_{k}}\right|_{\mathrm{BC}}=\delta_{1}(\varepsilon / 2) / 2 \quad \text { and } \quad\left|x^{k}(t)-\bar{u}(t)\right|_{X}<\delta_{1}(\varepsilon / 2) / 2 \quad \text { on }\left[t_{k}, s_{k}\right),
$$

where $\delta_{1}(\cdot)$ is the number given in Corollary 1. Taking a subsequence if necessary, we can assume that $\left(\bar{u}^{s_{k}}, F^{s_{k}}\right) \rightarrow(\bar{w}, P)$ compactly. We note that $X_{0}:=X_{\bar{u}, R^{+}} \cup$ 
$\overline{\left\{x_{t}^{k}: t_{k} \leq t \leq r_{k}, k \in N\right\}}$ is compact by Lemma 2 and that $\bar{u}(t)$ is asymptotically almost periodic by Theorem 1 . Since $s_{k} \rightarrow \infty$ as $k \rightarrow \infty$, it follows that $(\bar{w}, P) \in \Omega(\bar{u}, F)$. There exists a $k_{0}(\varepsilon)>0$ such that if $k \geq k_{0}(\varepsilon)$, then

$$
\rho\left(\bar{u}_{s_{k}}, \bar{w}_{0}\right)<\delta_{1}(\varepsilon / 2) / 2
$$

and

$$
\left|F\left(t+s_{k}, \phi\right)-P(t, \phi)\right|_{X}+\left|h^{k}\left(t+s_{k}\right)\right|_{X}<\delta_{1}(\varepsilon / 2) \quad \text { on } R^{+} \times X_{0} .
$$

Since $\bar{w}(t)$ is BC-TS with $\delta_{1}(\cdot)$, it follows from (16) and (17) that $\left|\bar{u}\left(t+s_{k}\right)-\bar{w}(t)\right|_{X}<\varepsilon / 2$ for all $t \geq 0$ whenever $k \geq k_{0}(\varepsilon)$. Notice that $x^{k}\left(t+s_{k}\right)$ is a solution defined on $\left[0, r_{k}-s_{k}\right]$ of

$$
\frac{d u}{d t}=A u(t)+P\left(t, u_{t}\right)+F\left(t+s_{k}, x_{t+s_{k}}^{k}\right)+h^{k}\left(t+s_{k}\right)-P\left(t, x_{t+s_{k}}^{k}\right),
$$

and

$$
\begin{aligned}
\rho\left(x_{s_{k}}^{k}, \bar{w}_{0}\right) & \leq \rho\left(x_{s_{k}}^{k}, \bar{u}_{s_{k}}\right)+\rho\left(\bar{u}_{s_{k}}, \bar{w}_{0}\right) \\
& \leq\left|x_{s_{k}}^{k}-\bar{u}_{s_{k}}\right|_{\mathrm{BC}}+\rho\left(\bar{u}_{s_{k}}, \bar{w}_{0}\right)<\delta_{1}(\varepsilon / 2) \quad \text { if } k \geq k_{0}(\varepsilon) .
\end{aligned}
$$

Therefore, if $k \geq k_{0}(\varepsilon)$, then

$$
\left|x^{k}\left(r_{k}\right)-\bar{w}\left(r_{k}-s_{k}\right)\right|_{X}<\varepsilon / 2,
$$

and hence

$$
\left|x^{k}\left(r_{k}\right)-\bar{u}\left(r_{k}\right)\right|_{X} \leq\left|x^{k}\left(r_{k}\right)-\bar{w}\left(r_{k}-s_{k}\right)\right|_{X}+\left|\bar{w}\left(r_{k}-s_{k}\right)-\bar{u}\left(r_{k}\right)\right|_{X}<\varepsilon / 2+\varepsilon / 2=\varepsilon,
$$

which contradicts to $\left|x^{k}\left(r_{k}\right)-\bar{u}\left(r_{k}\right)\right|_{X}=\varepsilon$. Consequently, $\bar{u}(t)$ must be eventually BC-TS. Since $\bar{u}(t)$ is unique for the initial value problem, Proposition 2 implies BC-TS of $\bar{u}(t)$.

By using (A2) directly for the convergence of $\bar{u}\left(t+s_{m}\right)$, the following theorem is shown by arguments parallel to those in the proof of Theorem 3; so we omit the proof.

THEOREM 4. Assume that $\mathcal{B}$ is a fading memory space and $\bar{u}(t)$ is unique for the initial value problem. If Equation (2) admits a limiting equation (3) whose solution $\bar{v}(t)$ such that $(\bar{v}, G) \in \Omega(\bar{u}, F)$ is $\mathcal{B}$-TS, then $\bar{u}(t)$ is $\mathcal{B}$-TS.

Next we will study the UAS property for Equation (2) via limiting equations. Before developing our argument, we emphasize that some additional condition must be imposed on (2) to get the result similar to Theorem 3 or Theorem 4 on the UAS property. Indeed, Kato's example (e.g. [11, pp. 143-145]) shows that there is an almost periodic differential equation with the property that zero is a UAS solution of the equation, but it admits a limiting equation whose zero solution is not UAS.

We say that Equation (2) is regular if each solution of equations in $H(F)$ is unique for the initial value problem. Under the regularity condition on (2), we will deduce a result on the uniform asymptotic stability. 
THEOREM 5. Assume that $\mathcal{B}$ is a fading memory space. If Equation (2) is regular and admits a limiting equation (3) whose solution $\bar{v}(t)$ such that $(\bar{v}, G) \in \Omega(\bar{u}, F)$ is BC-UAS, then $\bar{u}(t)$ is BC-UAS.

In order to prove the theorem, we need the following result.

Proposition 3. Assume that Equation (2) is regular. Then $\bar{u}(t)$ is BC-UAS if and only if any $\bar{v}(t)$ such that $(\bar{v}, G) \in \Omega(\bar{u}, F)$ is BC-UAS with a common triple $\left(\delta(\cdot), \delta_{0}, t_{0}(\cdot)\right)$. Furthermore, if $\bar{u}(t)$ is BC-UAS, then it is BC-TS.

PROOF. We will prove the "only if" part of the proposition. Let $\left(\delta(\cdot), \delta_{0}, t_{0}(\cdot)\right)$ be the triple for the BC-UAS of $\bar{u}(t)$, where we may assume $\delta_{0}<\delta(1)$. We first establish that

$$
\begin{aligned}
& (\bar{v}, G) \in \Omega(\bar{u}, F) \text { and }\left|\phi-\bar{v}_{\sigma}\right|_{\mathrm{BC}}<\delta(\eta / 2) \text { imply } \\
& |u(t, \sigma, \phi, G)-\bar{v}(t)|_{X}<\eta \quad \text { for } t \geq \sigma .
\end{aligned}
$$

Select a sequence $\left\{\tau_{k}\right\}$ with $\tau_{k} \rightarrow \infty$ as $k \rightarrow \infty$ such that $\left(\bar{u}^{\tau_{k}}, F^{\tau_{k}}\right) \rightarrow(\bar{v}, G)$ compactly, and consider any solution $u\left(\cdot, \sigma+\tau_{k}, \phi-\bar{v}_{\sigma}+\bar{u}_{\sigma+\tau_{k}}, F\right)$. For any $k \in N$, we set $x^{k}(t)=$ $u\left(t+\tau_{k}, \sigma+\tau_{k}, \phi-\bar{v}_{\sigma}+\bar{u}_{\sigma+\tau_{k}}, F\right)$. Since the solution $\bar{u}(t)$ of (2) is BC-US, from the fact that $\left|x_{\sigma}^{k}-\bar{u}_{\sigma+\tau_{k}}\right|_{\mathrm{BC}}=\left|\phi-\bar{v}_{\sigma}+\bar{u}_{\sigma+\tau_{k}}-\bar{u}_{\sigma+\tau_{k}}\right|_{\mathrm{BC}}=\left|\phi-\bar{v}_{\sigma}\right|_{\mathrm{BC}}<\delta(\eta / 2)$ it follows that

$$
\left|x^{k}(t)-\bar{u}\left(t+\tau_{k}\right)\right|_{X}<\eta / 2 \text { for all } t \geq \sigma \text { and } k \in N \text {; }
$$

hence $\sup \left\{\left|x_{t}^{k}\right|_{\mathcal{B}} \mid t \geq \sigma, k \in N\right\} \leq K\left(\eta / 2+|\bar{u}|_{[0, \infty)}\right)+M\left|\phi-\bar{v}_{\sigma}+\bar{u}_{\sigma+\tau_{k}}\right|_{\mathcal{B}}<\infty$ by (A1-iii). By Lemma 2 together with the same argument as in the proof of Proposition 2, we may assume that $x^{k}(t) \rightarrow y(t)$ as $k \rightarrow \infty$ uniformly on any compact set in $[\sigma, \infty)$ for some function $y:[\sigma, \infty) \mapsto X$. Since $x^{k}(\sigma)=\phi(0)-\bar{v}(\sigma)+\bar{u}\left(\sigma+\tau_{k}\right)$, we obtain $y(\sigma)=\phi(0)$. Hence, if we extend the function $y$ by setting $y_{\sigma}=\phi$, then $y \in C(R ; X)$ and $\left|x_{t}^{k}-y_{t}\right|_{\mathcal{B}} \rightarrow 0$ as $k \rightarrow \infty$ uniformly on any compact set in $[\sigma, \infty)$. Letting $k \rightarrow \infty$ in the equation

$$
x^{k}(t)=T(t-\sigma)\left\{\phi(0)-\bar{v}(\sigma)+\bar{u}\left(\sigma+\tau_{k}\right)\right\}+\int_{\sigma}^{t} T(t-s) F\left(s+\tau_{k}, x_{s}^{k}\right) d s, \quad t \geq \sigma,
$$

we obtain

$$
y(t)=T(t-\sigma) \phi(0)+\int_{\sigma}^{t} T(t-s) G\left(s, y_{s}\right) d s, \quad t \geq \sigma,
$$

which means that $y(t) \equiv u(t, \sigma, \phi, G)$ for $t \geq \sigma$ by the regularity assumption. Then (18) follows from (19) by letting $k \rightarrow \infty$.

Repeating the above arguments with $\eta=2$, one can establish that

$$
\begin{aligned}
& (\bar{v}, G) \in \Omega(\bar{u}, F) \text { and }\left|\phi-\bar{v}_{\sigma}\right|_{\mathrm{BC}}<\delta_{0} \text { imply } \\
& |u(t, \sigma, \phi, G)-\bar{v}(t)|_{X}<\varepsilon \text { for } t \geq \sigma+t_{0}(\varepsilon / 2) .
\end{aligned}
$$

Next we will prove the "if" part of the proposition. We first claim that $\bar{u}(t)$ is BC-US. If this claim is not true, then there are sequences $\left\{\tau_{n}\right\}, \tau_{n} \geq 0,\left\{r_{n}\right\}, r_{n}>0,\left\{\phi^{n}\right\} \subset \mathrm{BC}$, and solutions $\left\{u\left(t, \tau_{n}, \phi^{n}, F\right)\right\}$ and a constant $\varepsilon, 0<\varepsilon<\delta_{0}$, such that

$$
\left|\phi^{n}-\bar{u}_{\tau_{n}}\right| \mathrm{BC}<\frac{1}{n}
$$


and

(21) $\left|x^{n}\left(\tau_{n}+r_{n}\right)-\bar{u}\left(\tau_{n}+r_{n}\right)\right|_{X}=\varepsilon \quad$ and $\quad\left|x^{n}(t)-\bar{u}(t)\right|_{X}<\varepsilon \quad$ on $\left(-\infty, \tau_{n}+r_{n}\right)$,

where $x^{n}(t)=u\left(t, \tau_{n}, \phi^{n}, F\right)$. Consider the case where $r_{n} \rightarrow \infty$ as $n \rightarrow \infty$, and let $t_{0}=t_{0}(\varepsilon / 2)$. By the same reasoning as in the proof of Proposition 2, we may assume that there are a function $Q(t, \phi) \in \Omega(F)$ and solutions $\bar{v}(t)$ and $y(t)$ of

$$
\frac{d u}{d t}=A u(t)+Q\left(t, u_{t}\right)
$$

defined on $\left[0, t_{0}\right]$ such that $\left(\bar{u}^{\tau_{n}+r_{n}-t_{0}}, F^{\tau_{n}+r_{n}-t_{0}}\right) \rightarrow(\bar{v}, Q)$ compactly and $x^{n}\left(\tau_{n}+r_{n}-\right.$ $\left.t_{0}+t\right) \rightarrow y(t)$ uniformly on any compact set in $\left(-\infty, t_{0}\right]$ as $n \rightarrow \infty$. Letting $n \rightarrow \infty$ in $(21)$, we obtain that

$$
\left|y\left(t_{0}\right)-\bar{v}\left(t_{0}\right)\right|_{X}=\varepsilon \quad \text { and } \quad|y(t)-\bar{v}(t)|_{X} \leq \varepsilon<\delta_{0} \quad \text { for all } t \leq t_{0} .
$$

Thus $\left|y_{0}-\bar{v}_{0}\right|_{\mathrm{BC}}<\delta_{0}$, and hence it follows from the BC-UAS of $\bar{v}(t)$ that

$$
\left|y\left(t_{0}\right)-\bar{v}\left(t_{0}\right)\right|_{X}<\varepsilon / 2,
$$

which is a contradiction. Therefore, we can assume that $r_{n} \rightarrow r<\infty$ as $n \rightarrow \infty$.

By almost the same reasoning as in the preceding paragraph, one can deduce by (20) that the sequence $\left\{\tau_{n}\right\}$ is bounded. Hence we can assume $\tau_{n} \rightarrow \tau<\infty$ as $n \rightarrow \infty$. Moreover, we can assume that $x^{n}\left(\tau_{n}+t\right) \rightarrow x(t)$ uniformly on any compact set in $(-\infty, r)$ as $n \rightarrow \infty$, for some continuous function $x$ with $x_{0}=\bar{u}_{\tau}$. Observe that $x(t-\tau)$ is a solution of (2) defined on $[\tau, \tau+r)$; hence it follows from the uniqueness of $\bar{u}(t)$ for the initial value problem that $x(t) \equiv \bar{u}(\tau+t)$ for $t \in[0, r)$. By virtue of (20) and (21), one can choose $\tilde{r}_{n} \in\left(0, r_{n}\right)$ so that

$$
\left|x^{n}\left(\tau_{n}+\tilde{r}_{n}\right)-\bar{u}\left(\tau_{n}+\tilde{r}_{n}\right)\right|_{X}=\varepsilon / 2
$$

for $n \in N$. By the same reasoning as in the proof of Proposition 2, we see that $\inf _{n}\left(r_{n}-\tilde{r}_{n}\right)>$ 0 . Therefore it follows that $x\left(\tilde{r}_{n}\right)=\bar{u}\left(\tau+\tilde{r}_{n}\right)$, and

$$
\begin{aligned}
\varepsilon / 2 & =\left|x^{n}\left(\tau_{n}+\tilde{r}_{n}\right)-\bar{u}\left(\tau_{n}+\tilde{r}_{n}\right)\right|_{X} \\
& \leq\left|x^{n}\left(\tau_{n}+\tilde{r}_{n}\right)-x\left(\tilde{r}_{n}\right)\right|_{X}+\left|\bar{u}\left(\tau+\tilde{r}_{n}\right)-\bar{u}\left(\tau_{n}+\tilde{r}_{n}\right)\right|_{X} \rightarrow 0
\end{aligned}
$$

as $n \rightarrow \infty$, a contradiction. Consequently, $\bar{u}(t)$ must be BC-TS.

To establish the BC-UAS of $\bar{u}(t)$, it is sufficient to show that for any $\varepsilon>0$ there exists a $t_{0}(\varepsilon)>0$ such that $\left|\phi-\bar{u}_{\tau}\right|_{\mathrm{BC}}<\delta_{1}:=\delta\left(\delta_{0} / 2\right)$ implies $|u(t, \tau, \phi, F)-\bar{u}(t)|_{X}<\varepsilon$ for all $t \geq \tau+t_{0}(\varepsilon)$, where $\delta(\cdot)$ is the one ensured by the BC-US of $\bar{u}(t)$. If this is not true, then there exists an $\varepsilon>0$ and sequences $\left\{s_{n}\right\} \subset R^{+},\left\{t_{n}\right\}, t_{n} \rightarrow \infty$ as $n \rightarrow \infty,\left\{\phi^{n}\right\} \subset \mathrm{BC}$, and solutions $\left\{u\left(t, s_{n}, \phi^{n}, F\right)\right\}$ such that

$$
\left|\phi^{n}-\bar{u}_{s_{n}}\right|_{\mathrm{BC}}<\delta_{1}
$$

and

$$
\left|u\left(t_{n}+s_{n}, s_{n}, \phi^{n}, F\right)-\bar{u}\left(t_{n}+s_{n}\right)\right|_{X} \geq \varepsilon
$$


for all $n \in N$. It follows from (22) and the BC-US of $\bar{u}(t)$ that

$$
\left|u\left(t, s_{n}, \phi^{n}, F\right)-\bar{u}(t)\right|_{X}<\delta_{0} / 2 \text { for all } t \geq s_{n} .
$$

Set $z^{n}(t)=u\left(t+s_{n}+t_{n}-t_{0}, s_{n}, \phi^{n}, F\right)$ for $t \in R$, and let $t_{0}=t_{0}(\varepsilon)$. It follows from (24) that

$$
\left|z^{n}(t)-\bar{u}\left(t+s_{n}+t_{n}-t_{0}\right)\right|_{X}<\delta_{0} / 2 \text { for all } t \in\left[t_{0}-t_{n}, \infty\right) .
$$

Since $\sup \left\{\left|z^{n}(s)\right|_{X}: s \geq t_{0}-t_{n}, n \in N\right\}<\infty$ by (25), and since

$$
\begin{aligned}
z^{n}(t)= & T(1) z^{n}(t-1)+\int_{t-1}^{t} T(t-s) F\left(s+s_{n}+t_{n}-t_{0}, z_{s}^{n}\right) d s \\
= & T(1) z^{n}(t-1)+T(v) \int_{t-1}^{t-v} T(t-v-s) F\left(s+s_{n}+t_{n}-t_{0}, z_{s}^{n}\right) d s \\
& +\int_{t-v}^{t} T(t-s) F\left(s+s_{n}+t_{n}-t_{0}, z_{s}^{n}\right) d s
\end{aligned}
$$

for all $t \geq t_{0}+1-t_{n}$ and $v \in(0,1)$, by almost the same argument as in the proof of Lemma 2 and Proposition 2, we see that the set $\left\{z^{n}(t): t_{0}+1-t_{n} \leq t<\infty, n \in N\right\}$ is relatively compact in $X$ and that $\left\{z^{n}(t)\right\}$ is a family of equicontinuous on $\left[t_{0}+1-t_{n}, \infty\right)$. Applying the Ascoli-Arzéla theorem and the diagonalization procedure, one may assume that $z^{n}(t) \rightarrow z(t)$ uniformly on any compact set in $R$ for some bounded continuous function $z(t) \in C(R ; X)$. Then it follows from (A2) and (A1-iii) that $z_{t}^{n} \rightarrow z_{t}$ uniformly on any compact set in $R$. We may assume that $\left(\bar{u}^{s_{n}+t_{n}-t_{0}}, F^{s_{n}+t_{n}-t_{0}}\right) \rightarrow(\bar{v}, P)$ compactly for some $(\bar{v}, P) \in \Omega(\bar{u}, F)$. We note that $z(t)$ is a solution of

$$
\frac{d u}{d t}=A u(t)+P\left(t, u_{t}\right) .
$$

It follows from (25) that $|z(t)-\bar{v}(t)|_{X} \leq \delta_{0} / 2$ on $R$. In particular, $\left|z_{0}-\bar{v}_{0}\right|_{\mathrm{BC}} \leq \delta_{0} / 2<\delta_{0}$. Then $\left|z\left(t_{0}\right)-\bar{v}\left(t_{0}\right)\right|_{X}<\varepsilon$ by the BC-UAS of $\bar{v}(t)$. On the other hand, letting $n \rightarrow \infty$ in (23), we have $\varepsilon \leq\left|z\left(t_{0}\right)-\bar{v}\left(t_{0}\right)\right|_{X}$, which is a contradiction. Therefore $\bar{u}(t)$ must be BC-UAS.

The second part of Proposition 3 is a direct consequence of [8, proof of Theorem 2].

Proof OF THEOREM 5. Since Equation (2) is regular and $\bar{v}(t)$ is BC-UAS, any $\bar{w}(t)$ such that $(\bar{w}, E) \in \Omega(\bar{v}, G)$ is BC-UAS with a common $\left(\delta_{0}, \delta(\cdot), t_{0}(\cdot)\right)$ by Proposition 3. Therefore, by appling Proposition 3 again to $(\bar{v}, G)$, we see that $\bar{v}(t)$ is BC-TS, and hence $\bar{u}(t)$ is asymptotically almost periodic in $t$ by Theorem 1 . Since $\Omega(\bar{u}, F)=\Omega(\bar{v}, G)$, we have the conclusion by applying Proposition 3 .

Recall that the $\mathcal{B}$-stability property for the solution $\bar{u}(t)$ of (2) means that the difference $|u(t, \sigma, \phi, F)-\bar{u}(t)|_{X}$ remains small if $\left|\phi-\bar{u}_{\sigma}\right|_{\mathcal{B}}$ is small. If we estimate the difference of $u(t, \sigma, \phi, F)$ and $\bar{u}(t)$ as $\left|u_{t}(\sigma, \phi, F)-\bar{u}_{t}\right| \mathcal{B}$, we obtain another concept of the $\mathcal{B}$-stability property for $\bar{u}(t)$. The latter stability property is stronger than the former, in general. As stated in [7, Proposition 6.2.5], however, the two concepts of $\mathcal{B}$-stability property for $\bar{u}(t)$ are equivalent whenever $\mathcal{B}$ is a uniform fading memory space. Using this fact, one can establish the following theorem by a slight modification of the proof of Theorem 5 . 
THEOREM 6. Assume that $\mathcal{B}$ is a uniform fading memory space. If Equation (2) is regular and admits a limiting equation (3) whose solution $\bar{v}(t)$ such that $(\bar{v}, G) \in \Omega(\bar{u}, F)$ is $\mathcal{B}$-UAS, then $\bar{u}(t)$ is $\mathcal{B}$-UAS.

We emphasize that the condition that $\mathcal{B}$ is a uniform fading memory space cannot be removed in Theorem 6 . In fact, when $\mathcal{B}$ is a fading memory space which is not uniform, the $\mathcal{B}$-UAS property cannot necessarily be deduced from that of limiting equations even if the regularity condition is satisfied. In what follows, we shall provide an example which reflects the fact for the $\mathcal{B}$-UAS property.

Consider the following scalar equation

$$
\frac{d u}{d t}=-u(t)+\frac{u(0)}{1+t} .
$$

Equation (26) can be set up as a functional differential equation on the fading memory space $C_{g}^{0}(R)=: C_{g}^{0}$, where $g(s) \equiv 1+|s|$. Indeed, if we define a function $F$ by

$$
F(t, \phi)=-\phi(0)+\frac{\phi(-t)}{1+t}, \quad \phi \in C_{g}^{0},
$$

then the equation (26) is identical with (2) with $A=0$. For any $\phi \in C_{g}^{0}$, it follows that $\phi(-t) /(1+t) \rightarrow 0$ as $t \rightarrow \infty$. Hence we can see that $F(t, \phi)$ is asymptotically almost periodic in $t$ uniformly for $\phi \in C_{g}^{0}$, and the limiting equation of (26) is

$$
\frac{d v}{d t}=-v(t),
$$

whose zero solution is clearly $C_{g}^{0}$-UAS. On the other hand, the zero solution of (26) is not $C_{g}^{0}$-UAS. Indeed, for any $n \in N$, take a $\phi^{n} \in C_{g}^{0}$ such that $0 \leq \phi^{n}(s) \leq 1+|s|$ for all $s \leq 0$ with $\phi^{n}(0)=0$ and $\phi^{n}(-n)=1+n$. Then

$$
\begin{aligned}
u\left(t+n, n, \phi^{n}, F\right) & =\int_{n}^{t+n} e^{-(t+n-s)} \frac{\phi^{n}(-n)}{1+s} d s \\
& =\int_{0}^{t} e^{-(t-\tau)} \frac{1+n}{1+n+\tau} d \tau,
\end{aligned}
$$

and hence $\sup _{n \in N} u\left(n+t, n, \phi^{n}, F\right)=\int_{0}^{t} e^{-(t-\tau)} d \tau=1-e^{-t}$. Thus $\lim _{t \rightarrow \infty}\left[\sup _{n \in N} u(t+\right.$ $\left.\left.n, n, \phi^{n}, F\right)\right]=1$, which shows that the zero solution of (26) is not $C_{g}^{0}$-UAS because of the linearity of (26).

\section{REFERENCES}

[ 1 ] L. Amerio And G. Prouse, Almost-Periodic Functions and Functional Equations, Van Nostrand, New York, 1971.

[ 2 ] F. A. ATKINSON AND J. R. HADDOCK, On determing phase spaces for functional differential equations, Funkcial. Ekvac. 31 (1988), 331-347.

[ 3 ] P. Bondi, V. Moauro And F. Visentin, Limiting equations in the stability problem, Nonlinear Anal. 1 (1976/77), 123-128.

[ 4 ] A. D'AnNA, Total stability properties for an almost periodic equation by means of limiting equations, Funkcial. Ekvac. 27 (1984), 201-209. 
[ 5 ] H. R. HENRíQueZ, Periodic solutions of quasi-linear partial functional differential equations with unbounded delay, Funkcial. Ekvac. 37 (1994), 329-343.

[ 6 ] Y. HiNo AND S. MuRAKAmI, Total stability in abstract functional differential equations with infinite delay, Electron. J. Qual. Theory of Differ. Equ., Proc. 6'th Coll. QTDE, No. 13 (2000), 1-9.

[ 7 ] Y. Hino, S. Murakami and T. Naito, Functional Differential Equations with Infinite Delay, Lecture Notes in Math. 1473, Springer-Verlag, Berlin-Heidelberg, 1991.

[ 8 ] Y. Hino, S. Murakami and T. Yoshizawa, Stability and existence of almost periodic solutions for abstract functional differential equations with infinite delay, Tohoku Math. J. 49 (1997), 133-147.

[ 9 ] Y. HINO AND T. YoshizAWA, Total stability property in limiting equations for a functional differential equation with infinite delay, Časopis pro pěstování matematiky roč. 111 (1986), 62-69.

[10] V. Lakshmikantham and S. Leela, Nonlinear Differential Equations in Abstract Spaces, Pergamon Press, Oxford-New York, 1981.

[11] T. Yoshizawa, Stability Theory and the Existence of Periodic Solutions and Almost Periodic Solutions, Appl. Math. Sci. 14, Springer-Verlag, New York, 1975.

DEPARTMENT OF MATHEMATICS AND INFORMATICS CHIBA UNIVERSITY

1-33 YAYOICHO, INAGEKU, CHIBA 263-8522

JAPAN

E-mail address: hino@math.s.chiba-u.ac.jp
DEPARTMENT OF APPLIED MATHEMATICS

OKAYAMA UNIVERSITY OF SCIENCE

1-1 RIDAI-CHO, OKAYAMA 700-0005

JAPAN

E-mail address: murakami@youhei.xmath.ous.ac.jp 